\title{
Laser Based Spatial Spine Curve Determination in Scoliosis
}

\author{
Primož POREDOŠ* ${ }^{* 1}$, Dušan ČELAN ${ }^{2}$, Janez MOŽINA ${ }^{1}$, Matija JEZERŠEK ${ }^{1}$ \\ ${ }^{1}$ University of Ljubljana, Faculty of Mechanical Engineering, Ljubljana, Slovenia; \\ ${ }^{2}$ University Medical Centre Maribor, Maribor, Slovenia \\ http://dx.doi.org/10.15221/14.115
}

\begin{abstract}
We present the development of a novel method for the automatic determination of a spatial spine curve based on the measured 3D shape of the human back (automatic curve) with the 3D laser profilometer. The measuring system is based on a line laser triangulation, its measuring range at the distance of $1 \mathrm{~m}$ is $300 \times 700 \times 500 \mathrm{~mm}$, the measurement takes about 10 seconds and the single point measurement accuracy is $0.1 \mathrm{~mm}$. The method allows us to compare an automatically determined spatial spine curve with a spatial curve, determined by the physician (reference curve) with the method of root mean square deviation (RMSD) in the frontal and sagittal plane. To validate the method both automatic and reference curve were compared on one subject in three different upright postures with arms in positions: 1. arms released beside the body, 2 . upper arm in the horizontal position and forearm in vertical position upwards, 3. arms vertically at full stretch upwards. The results showed that the repeatability of the presented method for all three postures in the frontal and sagittal planes was $0.9 \mathrm{~mm}$ and $0.2 \mathrm{~mm}$, respectively, thus allowing to assess a valid quantitative analysis of spine curve course on the surface of the human back regardless of the human upright posture.
\end{abstract}

Keywords: Laser profilometry, 3D, back shape analysis, scoliosis, spatial spine curve, repeatability

\section{Introduction}

Laser measuring systems are becoming more and more widespread in industry and medicine. Especially in medicine, the occurrence of advanced therapies increases the need for non-contact, mobile, robust, accurate, effective and non-invasive measuring systems, which present minimal level of psychophysical stress for the patient [1,2]. This kind of metrology promises a possible avoidance of using invasive radiographic imaging methods, such as magnetic resonance imaging (MRI) and radiography (X-rays, CT). Currently, in modern medical practice there are often used numerous invasive and demanding psychophysical diagnostics [3-5].

As a result of the aforementioned disadvantages, we present a method that has a possibility of non-contact, accurate, quick and non-invasive $3 \mathrm{D}$ image acquisition of the human torsos with developed scoliosis as well as evaluation of the patient's body posture with the custom designed software. Incorrect posture is one of the reasons for a number of pathological conditions, for example scoliosis [6]. It is an abnormal 3D curvature of the vertebral column, manifested by elemental deformities in the three main anatomical planes: lateral curvature in frontal, anteroposterior deviation in sagittal, and vertebral axial rotation in the transverse plane [7, 8].

During human evolution the upright posture required adjustments of the spine, which is why the so-called double "S" shaped sagittal curvatures have developed. An anatomical expression of the curvatures is just one of the measures for a correct body posture in clinical assessment [9]. Physicians often try to determine the expression of the curvatures, which is often prone to subjective judgment of the particular physician. In addition to clinical assessments it is also important to quantitatively assess the curvatures using one of the imaging methods. For instance, radiographic imaging method is most accurate though, but it is known to be harmful to patients due to its cumulative effect of ionizing radiation [10]. Moreover, the MRI imaging method despite its accuracy is known to be expensive and hardly available [11]. Consequently, much efforts have been focused on alternative measuring methods for the spine curvatures assessment. A vast number of torso surface measurement methods are used in clinical assessment, most commonly mechanical methods such as the DeBrunner kyphometer [12], "Flexicurve ruler" [13], Gravity goniometer or inclinometer [14] and Myrin inclinometer [15], and optical imaging methods such as raster stereography [16], Moiré topography [17] and laser triangulation [7]. The latter method, which was used in the research presented in this paper, presents a completely non-invasive way of 2D and 3D acquisition of the patient's back and further analysis of the $3 \mathrm{D}$ surface of the body.

Thus, we propose a new, noninvasive and quick method for the automatic spatial determination of the spine curve of thoracic and lumbar spine based on the 3D shape measurement of the human torsos with developed scoliosis. Special emphasis in this paper is placed firstly on validation of the presented method by manual determination of the spine curve, and secondly on repeatability assessment of three different human upright postures

\footnotetext{
* primoz.poredos@fs.uni-lj.si; +386 14771 170; http://lab.fs.uni-lj.si/kolt
} 


\section{Methods}

The main objective of the 3D analysis was to automatically obtain spatial curve (automatic curve) of thoracic and lumbar spine based on the 3D shape measurement of the human torso. The newly developed algorithms allow us to analyze spine curves in the frontal $(X-Y)$ and sagittal $(X-Z)$ anatomical planes (Fig. 1). For the purpose of method validation, the manual determination of the spine curve (reference curve) on patient was carried out by palpation of the thoracic and lumbar spinous processes which were marked on the skin with a dark alcohol marker. An example of marked human back is shown in Fig. 3a. According to data in [18], the accuracy of palpation in the thoracic and lumbar spine zones was found to be $9.8 \mathrm{~mm}$.

Special attention was placed to repeatability assessment of three different human upright postures. The repeatability assessment was made due to patient's postural sway movements since average acquisition time of the back is about 10 seconds. This assessment also passed us an important information about the course of the spine curve, because different upright postures activate different muscles and and consequently there are notable differences on the relief of the back.

A clinically confirmed healthy male subject (age 54, height $167 \mathrm{~cm}$, body mass index 20.4) was scanned using the 3D laser profilometer ten times per each posture. The measurement protocol was as follows: subject was measured in the upright standing posture. During each measurement the subject was leaning against foam attached to the wall and held his breath. The number of consecutive measurements for each posture is 10 . The subject had his arms in three different positions:

\section{$>$ Posture 1: arms released beside the body \\ $>$ Posture 2: upper arm in the horizontal position, forearm in vertical position upwards \\ > Posture 3: arms vertically at full stretch upwards}

All measurements were performed at the University Medical Centre Maribor. The National Medical Ethics Committee of the Republic of Slovenia made a positive declaration and approved the realization of the presented research.

\subsection{Measuring system}

Three-dimensional measurements were performed using the 3D laser profilometer that is based on the translational scanning of a laser line across the measuring area. The principle of the measuring system is 3D laser triangulation [19]. A laser line projector generates one laser plane that is directed to the human back. The intersection of the laser plane with the measured surface presents an intersection curve, which is curved in accordance to the shape of the measured surface due to the known distance between the camera and laser projector $P$ and their mutual angle $\theta$. The position of the intersection curve in $3 \mathrm{D}$ space could then be calculated using the triangulation method. The principle of the laser triangulation is shown in Fig. 1.

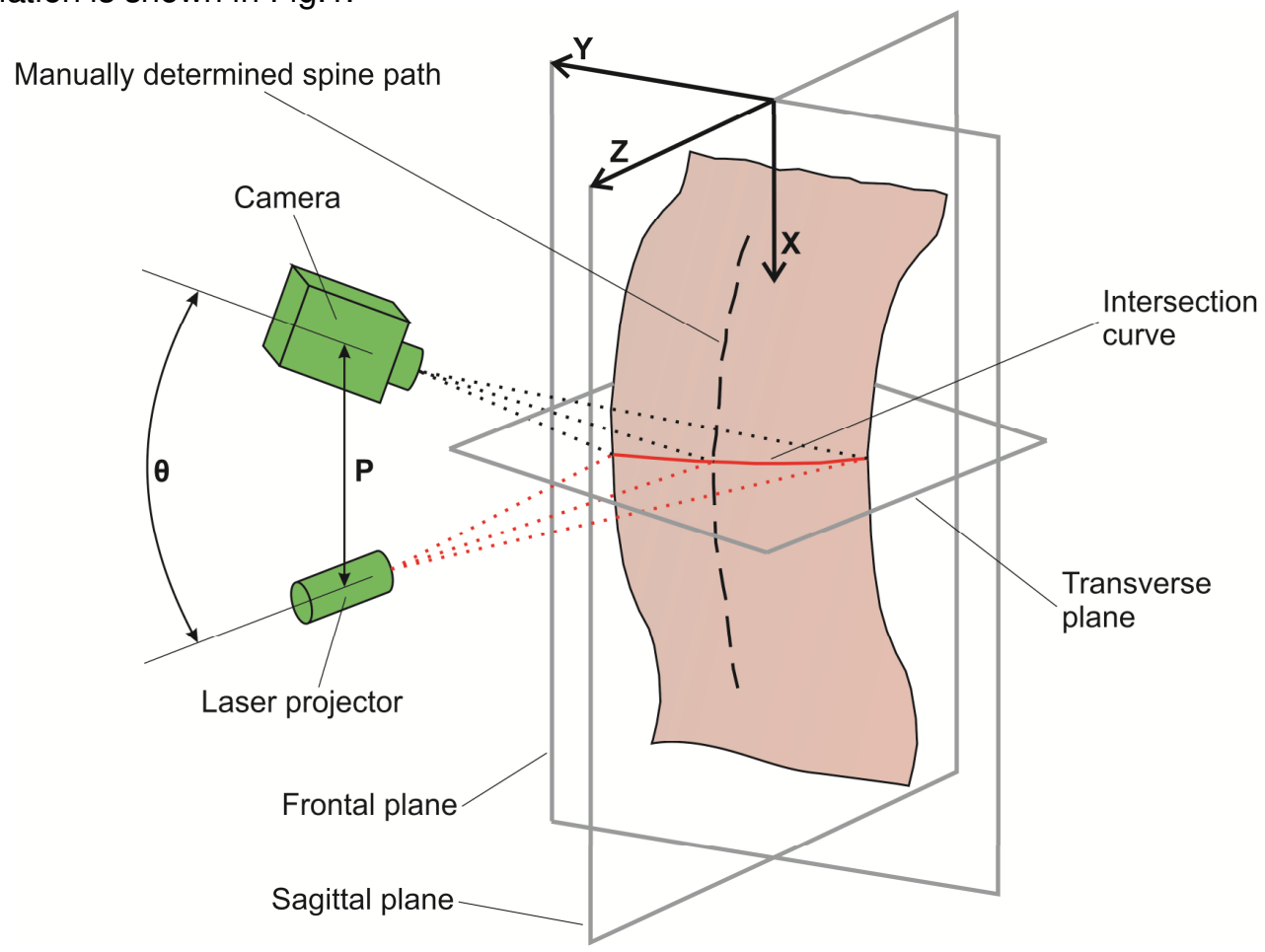

Fig. 1. Measuring system scheme. 
Two fundamental elements of the system are a grayscale camera and a laser line projector, as shown in Fig. 2. The camera (A301f, Basler, Ahrensburg, Germany) has a 1/2" CCD image sensor, a resolution of $658 \times 494$ pixels and a maximum frequency of video acquisition of $80 \mathrm{~Hz}$. The connection between the camera and PC was established via the FireWire bus. The laser line projector, with $5 \mathrm{~mW}$ of power and a wavelength $(\lambda)$ of $670 \mathrm{~nm}$ (red), generated one laser plane. Since we wanted to capture the human back, the assembly of the camera and laser projector had to be moved along the $\mathrm{X}$ direction and simultaneously the system had to acquire profiles of the measured surface. The assembly movement was performed using a computer-controlled linear translator (see Fig. 2).

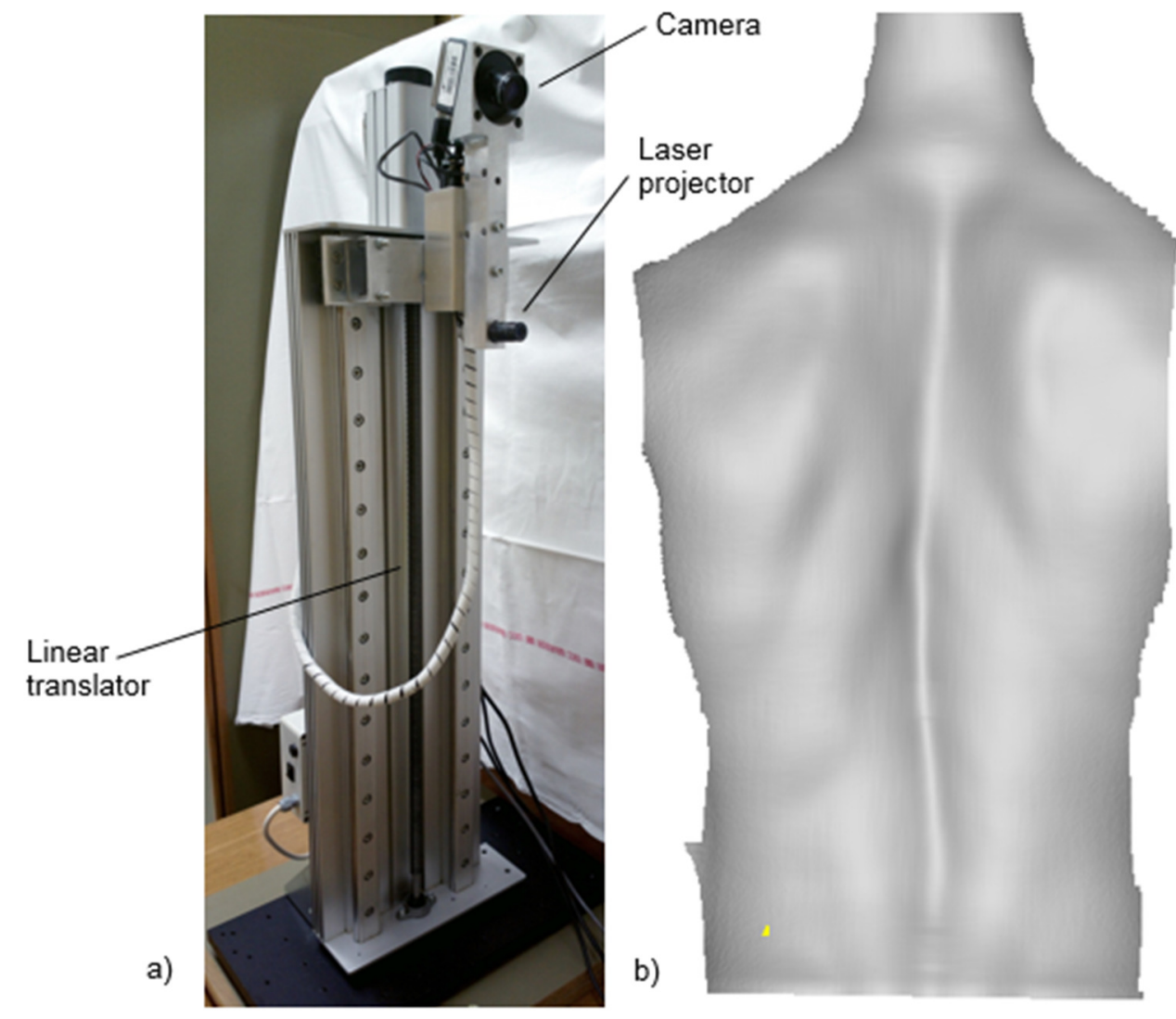

Fig. 2. Laser profilometer (a) for the 3D shape measurement of the human back (b).

The measurement resolution, i.e. a minimum distance between two adjacent measured profiles, depended on the translation velocity and measurement frequency and was approximately $0.9 \mathrm{~mm}$. At a distance of $1 \mathrm{~m}$ the measuring range was $300 \times 700 \times 500 \mathrm{~mm}$ (width $\times$ height $\times$ depth). The accuracy of single point measurement was $0.1 \mathrm{~mm}$ after the calibration [20]. The measured surface, which was presented as an ordered point cloud, was stored after the measurement completion. Then, the corresponding spatial ( $\mathrm{X}, \mathrm{Y}$, and $\mathrm{Z}$ ) coordinates and brightness information (in an image coordinate system $u, v)$ for each point of intersection curve was stored.

\subsection{Measurement analysis}

\subsection{1 $2 \mathrm{D}$ reference curve determination}

After the region of interest $(\mathrm{ROI})$ was defined, the reference curve determination was performed on a grayscale image of the back that was previously filtered with a Gaussian convolution filter (kernel size: $7 \times 7$ points). The determination was based on searching of manual markings by identifying the minimum pixel intensity along individual image rows. With the appropriate settings (intensity threshold detection ranges from 0.08 to 0.15$)$ the dashed reference line was determined. Gaps between individual lines were filled with one-dimensional interpolation. Thus, a continuous 2D curve that represents the reference curve was obtained, which shows Fig. 3b. 


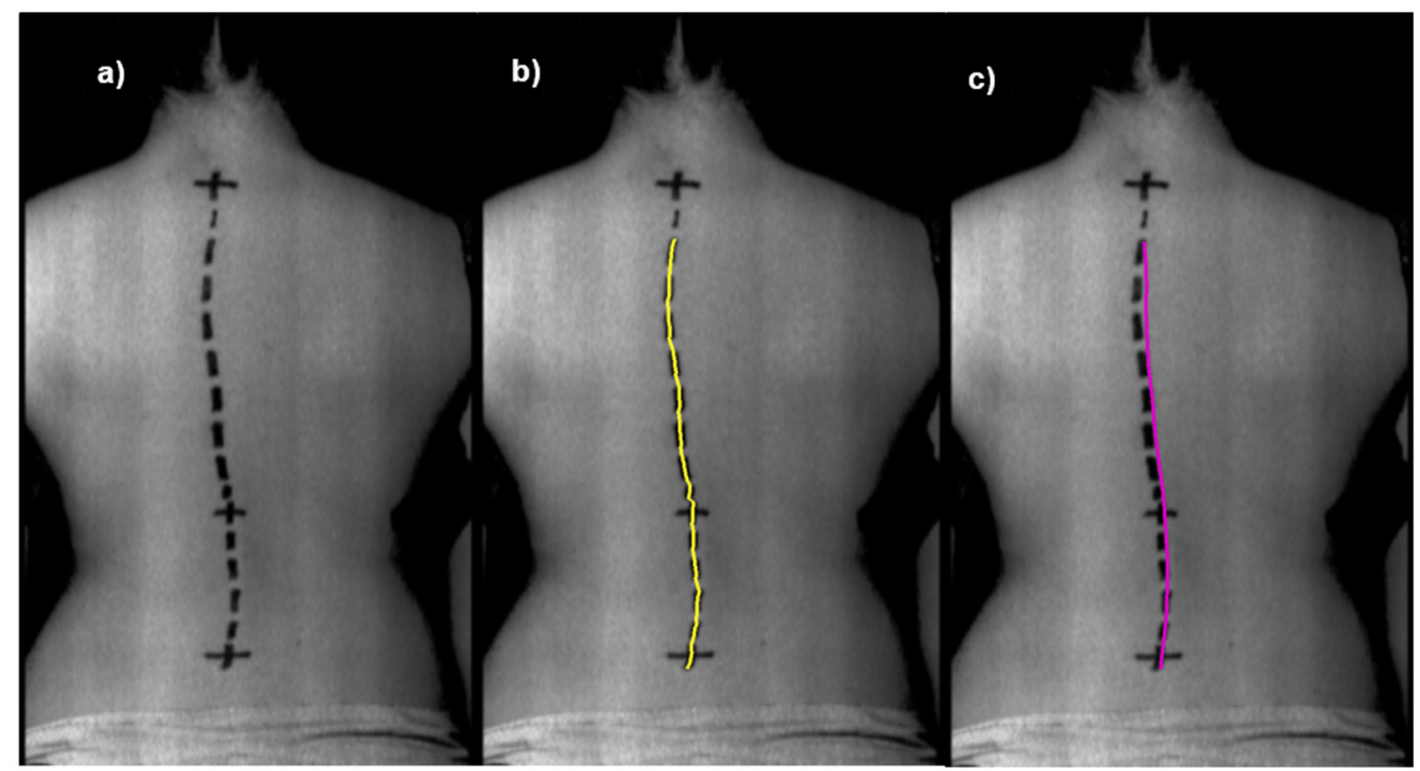

Fig. 3. Marked spinous processes (a), determined 2D reference curve (b), determined 3D automatic curve (c).

\subsubsection{D automatic curve determination}

For the purpose of automatic curve determination, the 3D depth image of a back was used. Therefore, surface curvature along the $Y$ axis was calculated from the depth image using the following equation:

$$
K=\frac{\frac{d^{2} Z}{d Y^{2}}}{\left[1+\left(\frac{d Z}{d Y}\right)^{2}\right]^{3 / 2}}
$$

Like before, the automatic curve determination was performed by searching the extremes of curvature along an individual image row of the calculated back surface curvature. The calculation is showed in Fig. 4. With the appropriate settings (intensity threshold detection set to zero) a continuous 2D curve that represents the automatic curve was obtained, which shows Fig. 3c.

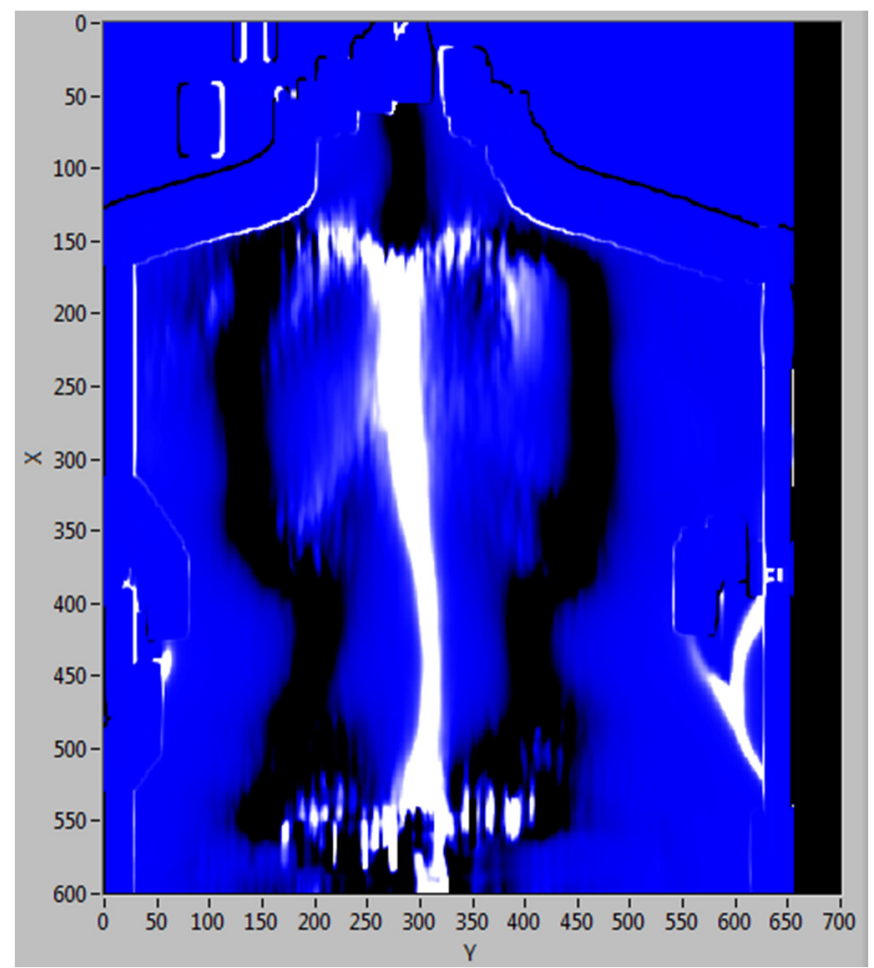

Fig. 4. Calculation of the back surface curvature. Positive curvature are represented as bright areas, while negative curvature are represented as darker areas. 


\subsubsection{Extraction of spatial curves}

The extraction of the spatial 3D reference and automatic curves was based on the previously detected $2 \mathrm{D}$ curves that were placed in an image coordinate system $u, v$. Thus, both reference and automatic curves that are represented by the $\mathrm{X}, \mathrm{Y}, \mathrm{Z}$ coordinates were obtained. An example of the automatic spatial spine curve overlying the visualized 3D shape of the back is shown in Fig. 5.

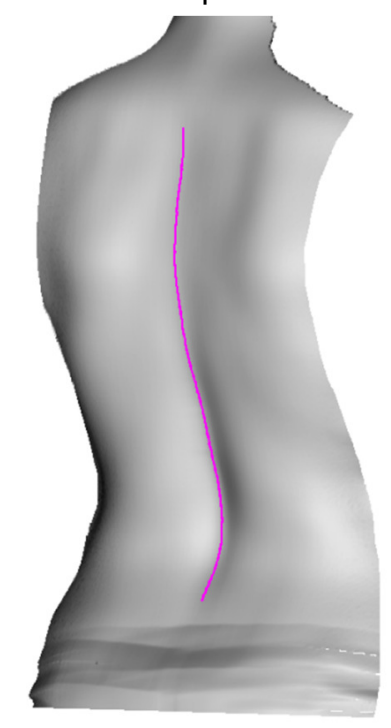

Fig. 5. Automatic spatial 3D spine curve.

\subsubsection{Calculation of root mean square deviation}

To compare the paths of the reference and automatic spatial spine curves, the root mean square deviation (RMSD) calculation was performed using following equations:

$$
\begin{aligned}
R M S D_{X-Y} & =\sqrt{\frac{1}{n} \sum_{i=1}^{N}\left(Y_{R, i}-Y_{A, i}\right)^{2}} \\
R M S D_{X-Z} & =\sqrt{\frac{1}{n} \sum_{i=1}^{N}\left(Z_{R, i}-Z_{A, i}\right)^{2}}
\end{aligned}
$$

where $\mathrm{R}$ denotes a reference curve and $\mathrm{A}$ denotes an automatic curve.

\section{Results}

The results of the reference and automatic spatial spine curve comparison for three different postures are presented in Table 1. Based on the average RMSD values, which are calculated on the basis of 10 successive measurements per each posture, we can conclude that the posture type has a minor effect on the overall overlapping of both curves for frontal and sagittal plane. The maximum value of the average RMSD also did not exceed the error of palpation mentioned in previous chapter $(3.36 \mathrm{~mm}<$ $9.8 \mathrm{~mm}$ ). However, we assume that the existing differences between the reference and automatic curves are due to different skin fold thickness across the subject's back and different spine curve expression with the positive and/or negative curvature of the back. The third reason could be the transverse movement of the skin in regard to the vertebrae after the manual marking.

Table 1. RMSD between reference and automatic spine curves for three different postures.

\begin{tabular}{c|c|c}
\hline Posture & $\mathrm{RMSD}_{\mathrm{X}-\mathrm{Y}}^{\mathrm{AVG}}[\mathrm{mm}]$ & $\mathrm{RMSD}_{\mathrm{X}-\mathrm{Z}}^{\mathrm{AVG}}[\mathrm{mm}]$ \\
\hline Posture 1 & 2.66 & 1.16 \\
\hline Posture 2 & 2.90 & 1.14 \\
\hline Posture 3 & 3.36 & 0.76 \\
\hline
\end{tabular}

Fourth reason could be linked to patient's postural sway movements since average acquisition time of the back is about 10 seconds. 
The repeatability results based on 10 successive measurements for three different postures are shown in Table 2 and Fig. 6, 7, 8. In the frontal plane a cluster of 10 lines representing the reference and automatic curves differences $\Delta \mathrm{Y}_{\mathrm{M}-\mathrm{A}}$ are shown in Figures 6-8a. A standard deviation of $\Delta \mathrm{Y}_{\mathrm{M}-\mathrm{A}}$ at each $\mathrm{X}$ coordinate $-\Delta \mathrm{Y}^{S D}{ }^{M-A}$ is represented with blue curve, while the standard deviation of 10 reference curve determinations $\mathrm{Y}_{\mathrm{M}}^{\mathrm{SD}}$ is represented with the black curve in Figures 6-8b. Similarly, in the sagittal plane a cluster of 10 lines representing the reference and automatic curves differences $\Delta Z_{M-A}$ are shown in Figures 6-8c. A standard deviation of $\Delta Z_{\mathrm{M}-\mathrm{A}}$ at each $\mathrm{X}$ coordinate $-\Delta \mathrm{Z}^{\mathrm{SD}}{ }_{\mathrm{M}-\mathrm{A}}$ is represented with blue curve, while the standard deviation of 10 reference curve determinations $Z^{S D}{ }_{M}$ is represented with the black curve in Figures 6-8b. Based on these results it can be noticed that posture variations $Y^{\mathrm{SD}, A V G}{ }_{M}$ and $\mathrm{Z}_{\mathrm{SD}, A V G}{ }_{\mathrm{M}}$ are notably higher in both the frontal and sagittal planes than the reference and automatic curve difference variations $\Delta Y^{S D, A V G}{ }_{M-A}$ and $\Delta Z^{S D, A V G}{ }_{M-A}$ for all three postures. We can conclude that in both the frontal and sagittal planes, the repeatability of the spine curve differences is much better than the repeatability of upright posture, regardless the position of the arms.

Table 2. Variation of the difference between the reference and automatic curves and average posture variations for three different postures.

\begin{tabular}{c|c|c|c|c}
\hline Posture & \multicolumn{2}{|c|}{ Frontal plane } & \multicolumn{2}{c}{ Sagittal plane } \\
\hline & $\Delta \mathrm{Y}^{\mathrm{SD}, \mathrm{AVG}}{ }_{\mathrm{M}-\mathrm{A}}[\mathrm{mm}]$ & $\mathrm{Y}^{\mathrm{SD}, \mathrm{AVG}}{ }_{\mathrm{M}}[\mathrm{mm}]$ & $\Delta \mathrm{Z}^{\mathrm{SD}, \mathrm{AVG}}{ }_{\mathrm{M}-\mathrm{A}}[\mathrm{mm}]$ & $\mathrm{Z}^{\mathrm{SD}, \mathrm{AVG}}{ }_{\mathrm{M}}[\mathrm{mm}]$ \\
\hline Posture 1 & 0.70 & 5.64 & 0.17 & 4.31 \\
\hline Posture 2 & 0.75 & 3.78 & 0.10 & 3.67 \\
\hline Posture 3 & 1.19 & 7.72 & 0.21 & 2.78 \\
\hline
\end{tabular}
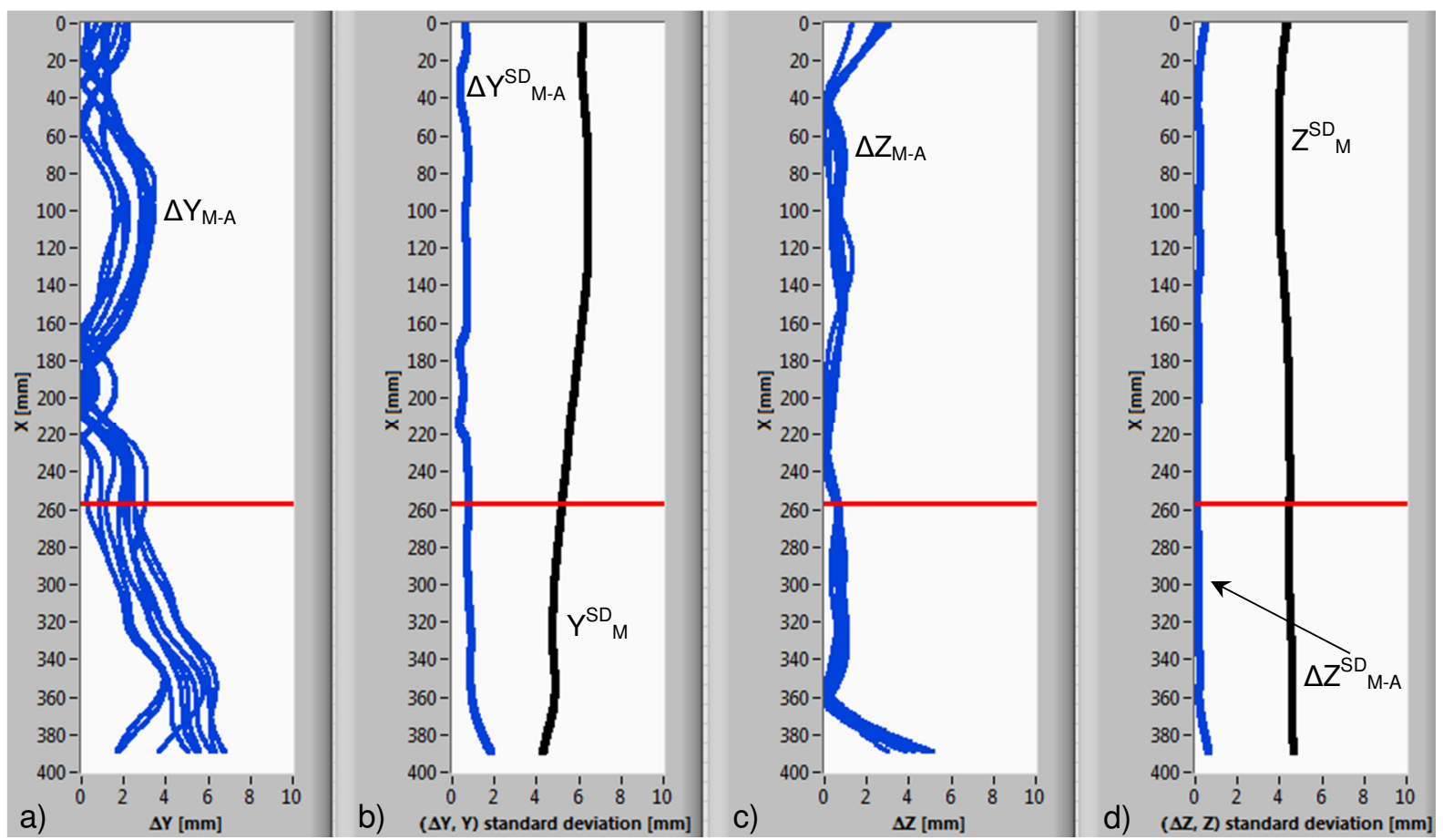

Fig. 6. Repeatability results for Posture 1. The difference between the reference and automatic curves in the frontal plane is represented by a cluster of 10 lines (a). Standard deviation of differences is represented by the blue curve and standard deviation of 10 reference curve determinations is represented by the black curve in the frontal plane

(b). The differences between the reference and automatic curves in the sagittal plane (c). Standard deviation of differences is represented by the blue curve and standard deviation of 10 reference curve determinations is represented by the black curve in the sagittal plane (d). The transition between the thoracic and lumbar spine zones is represented by the red line. Same description for the Fig. 7 and Fig. 8. 

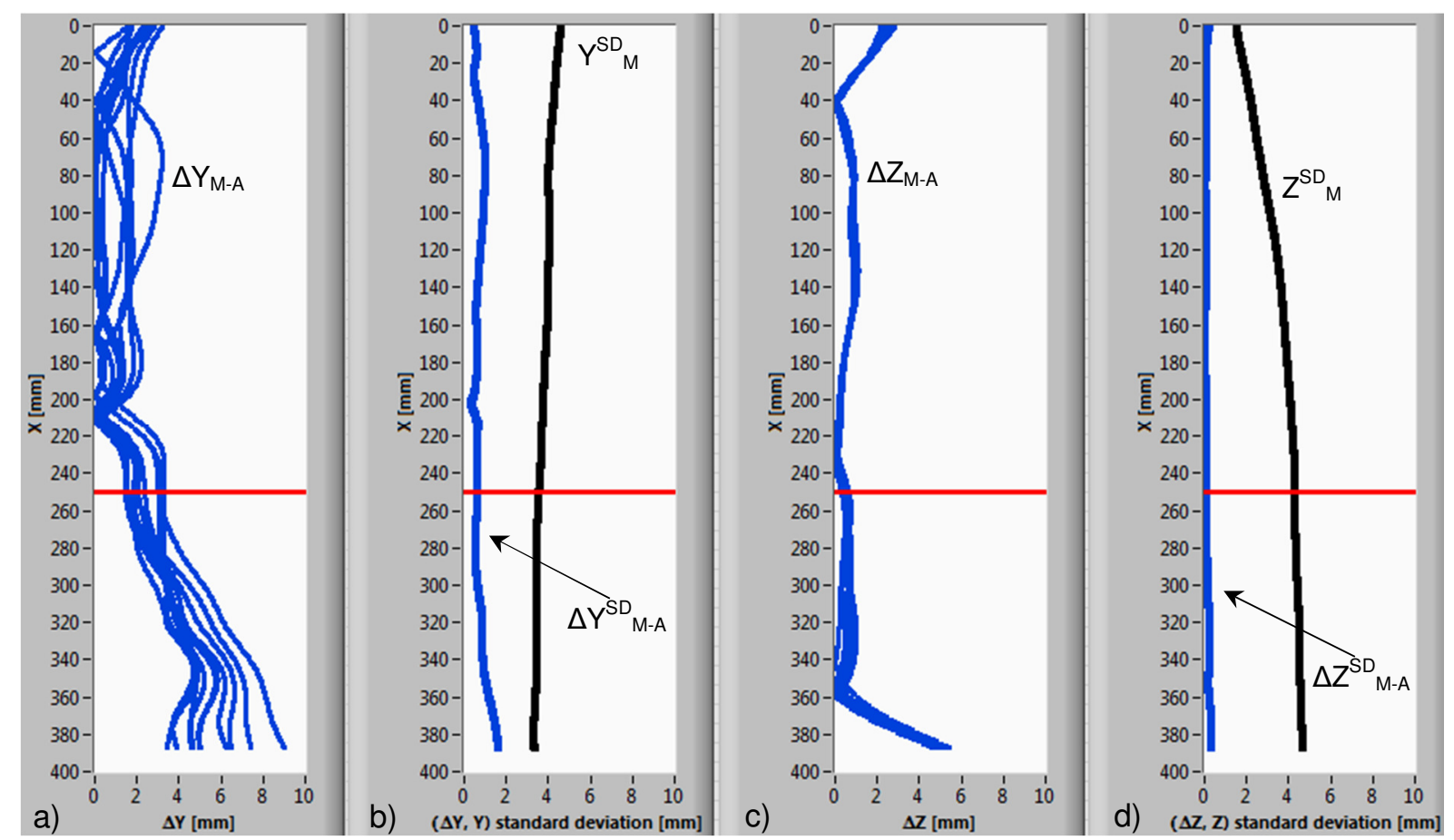

Fig. 7. Repeatability results for Posture 2.
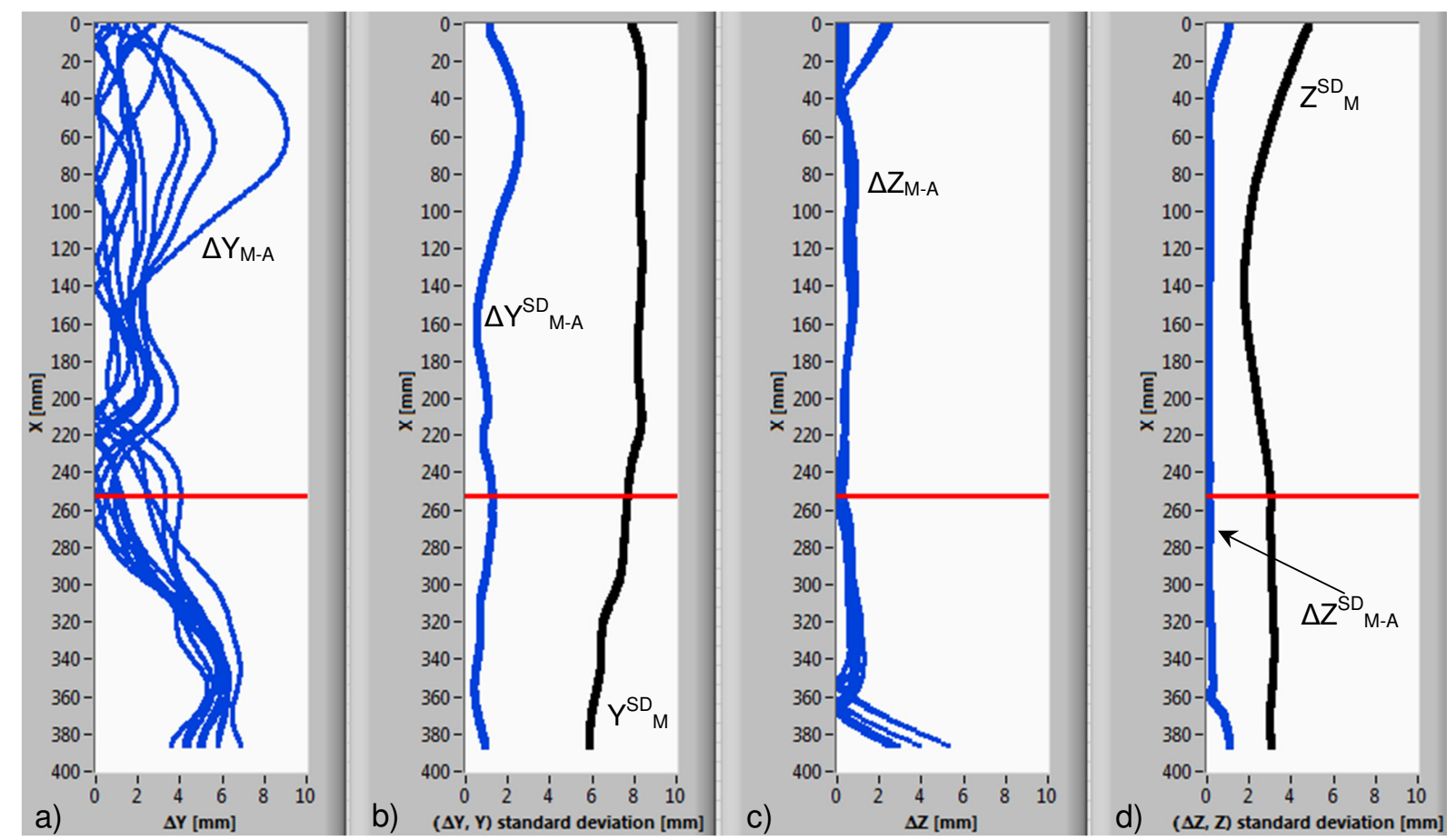

Fig. 8. Repeatability results for Posture 3.

Based on these results we can say that any of the presented postures is suitable for measurement analysis of the human back. The main difference between the presented postures is in the external shape of the back. If we look at Fig. 9 we can see that each of the postures has differently expressed determined automatic curves. In Fig. 9a the subject had arms released beside the body. It can be noticed that in the thoracic spine there is a huge change of curvature, from positive to negative, represented by the purple and turquoise blue curves. In Fig. 9b the subject had upper arm in the horizontal position and forearm in vertical position upwards. The change of curvature can be noticed lower than in previous case, most probable at the transition from the thoracic to lumbar part of the spine. However, in this case the purple line shows the best agreement with the manual markings. The purple line - automatic curve in Fig. 9c, when the subject had arms vertically at full stretch upwards, shows similar anomaly in the transition part of the spine, but in the thoracic part there is again a notable slight change in curvature. The purple line turns away from manual markings, while the turquois blue line is short, but has very good agreement with the manual marking. To conclude the observations from the Figure 9 and all previous results, we can say that there are not huge differences 
between the presented upright postures. We assume that every posture shows slightly different automatic spine curve not only for one subject. If we compare randomly chosen subjects in same position, every subject will show differently expressed relief of the back due to a different physical condition. However, according to 3-D scanning methodologies for internationally compatible anthropometric databases (ISO 20685:2010) that define four standard positions of the subject for obtaining reliable data, we propose the Posture 1 in which the subject was in standing upright posture with arms placed by the body as a recommended posture for 3D acquisitions of the human back. The Posture 1 is very similar to Standing position A defined in the aforementioned standard as: "The head is in the Frankfurt plane; the long axes of the feet should be parallel to one another and $200 \mathrm{~mm}$ apart; the upper arms are abducted to form a $20^{\circ}$ angle with the sides of the torso, and the elbows are straight; the palms face backward, and the subject is breathing quietly. This position may be used for obtaining circumferences of the upper and lower limbs" (CEN national Members, 2010, p.14).

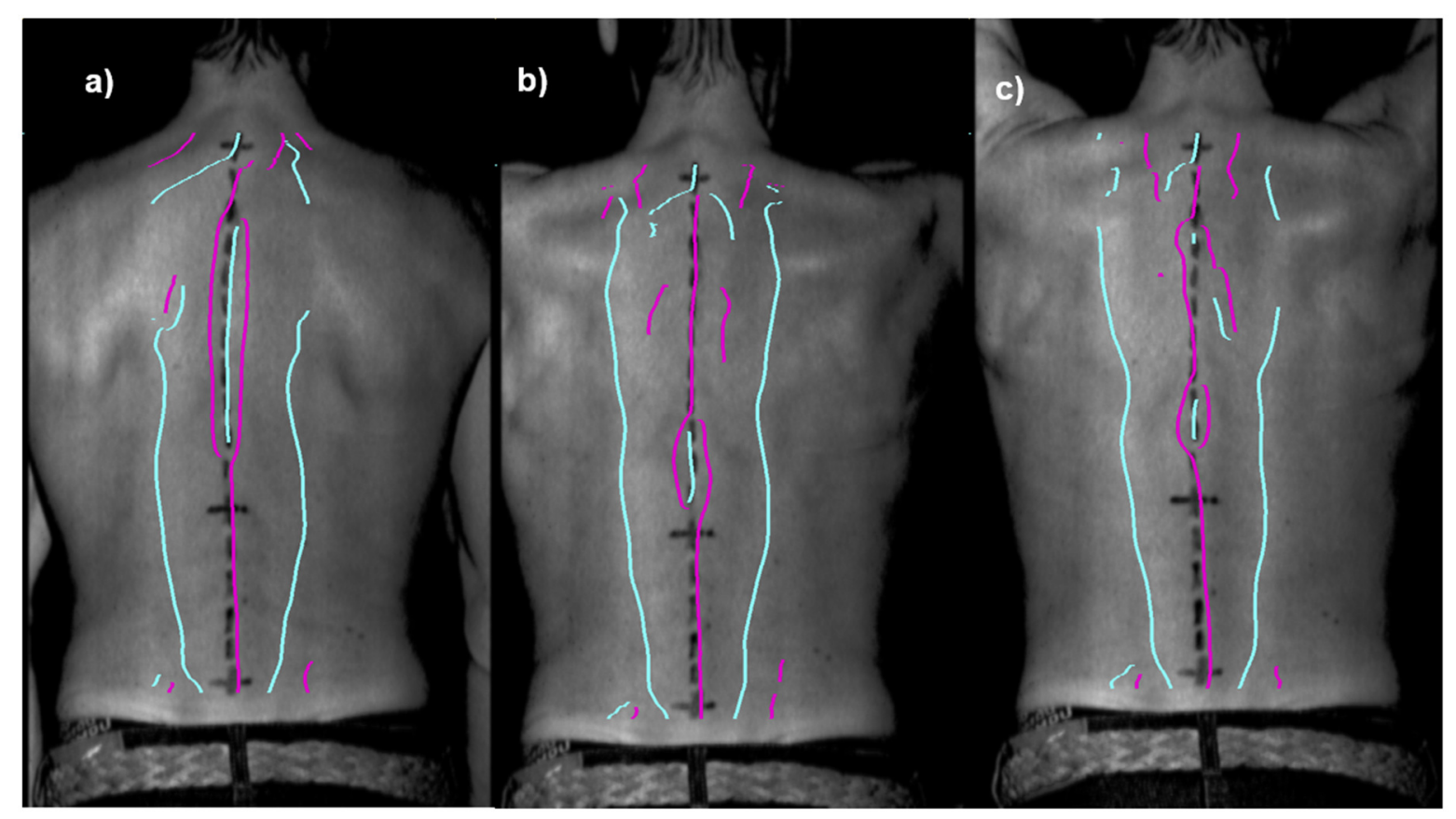

Fig. 9. Differently expressed determined automatic spine curves for three different human upright postures: Posture 1 (a), 2 (b) and 3 (c). Purple lines represent positive curvature, while turquoise blue lines represent negative curvature.

\section{Conclusions}

In this paper, we present a new, non-invasive, quick and accurate method for the 3D acquisition of the human back and automatic determination of human spine curve. Three-dimensional measurements of the backs were performed using the 3D laser profilometer with the single point measurement accuracy $0.1 \mathrm{~mm}$. The measurement took about 10 seconds.

Both static human upright posture as well as dynamic movements of the torso are subjected to specific curvatures and motion ability of the spine, which we wanted to quantify. A 3D acquisition of the back surface with many bulges and dips and measurement analysis of the acquired surface determined the line, where skin overlaid tips of the spinous processes. Although the human body is approximately symmetrical, there are several differences between the left and right side of the back, which are highly emphasized by some pathological conditions, for instance scoliosis. The 3D shape of the back is also influenced by different posture of the upper limbs. When torso in normal standing posture is being observed, arms are released beside the body. Raising upper limbs alters the anatomy of the back surface due to changes of sagittal curvatures of the spine, movements of the shoulder blade and activation of the dorsal subcutaneous muscles. In the presented study the automatic determination of the spinous processes path was conducted for three human upright postures. Comparison of automatically determined spine curve with reference spine curve, based on the palpatory determination of spinous processes, showed typical differences in the frontal and sagittal planes as $3.00 \mathrm{~mm}$ and $1.00 \mathrm{~mm}$, respectively.

The results of measurement analysis showed that the repeatability of the presented algorithms in the frontal and sagittal planes were $0.9 \mathrm{~mm}$ and $0.2 \mathrm{~mm}$, respectively. Therefore, we can say that the method of laser triangulation, upgraded with the presented algorithms, allows us a valid quantitative analysis of spine curve course on the surface of the human back regardless of the human upright posture. 


\section{References}

[1] M. Jezeršek, "High-speed measurement of foot shape based on multiple-laser-plane triangulation," Optical Engineering, vol. 48, p. 113604, 2009.

[2] K. Povšič, M. Fležar, J. Možina, and M. Jezeršek, "Laser 3-D measuring system and real-time visual feedback for teaching and correcting breathing," J Biomed Opt, vol. 17, p. 036004, Mar 2012.

[3] Y. Wang, B. Qian, B. Li, G. Qin, Z. Zhou, Y. Qiu, et al., "Metal artifacts reduction using monochromatic images from spectral CT: Evaluation of pedicle screws in patients with scoliosis," European Journal of Radiology, vol. 82, pp. e360-e366, 8// 2013.

[4] A. A. Musa, "Methods of assessing spinal radiographs in scoliosis are functions of its geometry," Computerized Medical Imaging and Graphics, vol. 23, pp. 201-203, 7// 1999.

[5] M. E. Alves de Araújo, E. Bezerra da Silva, D. Bragade Mello, S. A. Cader, A. Shiguemi Inoue Salgado, and E. H. M. Dantas, "The effectiveness of the Pilates method: Reducing the degree of non-structural scoliosis, and improving flexibility and pain in female college students," Journal of Bodywork and Movement Therapies, vol. 16, pp. 191-198, 4// 2012.

[6] J. L. P. do Rosário, "Photographic analysis of human posture: A literature review," Journal of Bodywork and Movement Therapies, vol. 18, pp. 56-61, 1// 2014.

[7] J. C. Rodríguez-Quiñonez, O. Y. Sergiyenko, L. C. B. Preciado, V. V. Tyrsa, A. G. Gurko, M. A. Podrygalo, et al., "Optical monitoring of scoliosis by 3D medical laser scanner," Optics and Lasers in Engineering, vol. 54, pp. 175-186, 3// 2014.

[8] M. M. Adankon, J. Dansereau, H. Labelle, and F. Cheriet, "Non invasive classification system of scoliosis curve types using least-squares support vector machines," Artificial Intelligence in Medicine, vol. 56, pp. 99-107, 10// 2012.

[9] D. Czaprowski, P. Pawłowska, Ł. Stoliński, and T. Kotwicki, "Active self-correction of back posture in children instructed with 'straighten your back' command," Manual Therapy.

[10] C. M. Ronckers, M. M. Doody, J. E. Lonstein, M. Stovall, and C. E. Land, "Multiple diagnostic X-rays for spine deformities and risk of breast cancer," Cancer Epidemiol Biomarkers Prev, vol. 17, pp. 605-13, Mar 2008.

[11] C. Bergeron, F. Cheriet, J. Ronsky, R. Zernicke, and H. Labelle, "Prediction of anterior scoliotic spinal curve from trunk surface using support vector regression," Engineering Applications of Artificial Intelligence, vol. 18, pp. 973-983, 12// 2005.

[12] D. M. Kado, L. Christianson, L. Palermo, R. Smith-Bindman, S. R. Cummings, and G. A. Greendale, "Comparing a supine radiologic versus standing clinical measurement of kyphosis in older women: the Fracture Intervention Trial," Spine (Phila Pa 1976), vol. 31, pp. 463-7, Feb 15 2006.

[13] K. M. Lundon, A. M. Li, and S. Bibershtein, "Interrater and intrarater reliability in the measurement of kyphosis in postmenopausal women with osteoporosis," Spine (Phila Pa 1976), vol. 23, pp. 1978-85, Sep 151998.

[14] B. S. Lonner, J. D. Auerbach, O. Boachie-Adjei, S. A. Shah, N. Hosogane, and P. O. Newton, "Treatment of thoracic scoliosis: are monoaxial thoracic pedicle screws the best form of fixation for correction?," Spine (Phila Pa 1976), vol. 34, pp. 845-51, Apr 152009.

[15] G. Ohlen, T. Wredmark, and E. Spangfort, "Spinal sagittal configuration and mobility related to low-back pain in the female gymnast," Spine (Phila Pa 1976), vol. 14, pp. 847-50, Aug 1989.

[16] L. Hackenberg, E. Hierholzer, W. Pötzl, C. Götze, and U. Liljenqvist, "Rasterstereographic back shape analysis in idiopathic scoliosis after posterior correction and fusion," Clinical Biomechanics, vol. 18, pp. 883-889, $12 / / 2003$.

[17] J. Legaye, "Follow-up of the sagittal spine by optical technique," Annals of Physical and Rehabilitation Medicine, vol. 55, pp. 76-92, 3// 2012.

[18] C. E. Aylott, R. Puna, P. A. Robertson, and C. Walker, "Spinous process morphology: the effect of ageing through adulthood on spinous process size and relationship to sagittal alignment," Eur Spine J, vol. 21, pp. 1007-12, May 2012.

[19] M.-C. Amann, T. Bosch, R. Myllyla", M. Rioux, and M. Lescure, "Laser ranging: a critical review of usual techniques for distance measurement," Optical Engineering, vol. 40, pp. 10-19, 2001.

[20] D. Čelan, M. Palfy, D. Bračun, Z. Turk, J. Možina, and R. Komadina, "Measurement of Spinal Sagittal Curvatures using the Laser Triangulation Method," Collegium Antropologicum, vol. 36, pp. 179-186, 2012. 